\title{
HIGH FREQUENCY MEASUREMENT OF TEMPERATURE AND COMPOSITION SPOTS WITH LITGS
}

\author{
Francesca De Domenico*1, Steven M. Lowe ${ }^{1}$, Luming Fan ${ }^{1}$, Simone Hochgreb ${ }^{1}$ \\ Priyav Shah ${ }^{2}$, Benjamin A.O. Williams ${ }^{2}$, Paul Ewart ${ }^{3}$ \\ ${ }^{1}$ Department of Engineering, University of Cambridge, UK \\ ${ }^{2}$ Department of Engineering, ${ }^{3}$ Department of Physics, University of Oxford, UK
}

\begin{abstract}
Temperature and composition spots in a turbulent flow are detected and time-resolved using Laser Induced Thermal Grating Spectroscopy (LITGS). A $355 \mathrm{~nm}$ wavelength PIV laser is operated at $0.5-1 \mathrm{kHz}$ to generate the thermal grating using biacetyl as an absorber in trace amounts. In a open laminar jet, a feasibility study shows that small $(\simeq 3 \%)$ fluctuations in the mean flow properties are well captured with LITGS. However, corrections of the mean flow properties by the presence of the trace biacetyl are necessary to properly capture the fluctuations. The actual density and temperature variation in the flow are determined using a calibration procedure validated using a laminar jet flow. Finally, travelling entropy and composition spots are directly measured at different locations along a quartz tube, obtaining good agreement with expected values. This study demonstrates that LITGS can be used as a technique to obtain instantaneous, unsteady temperature and density variations in a combustion chamber, requiring only limited optical access.
\end{abstract}

\section{NOMENCLATURE}

$c \quad$ Speed of sound $[\mathrm{m} / \mathrm{s}]$

$c_{p} \quad$ Specific heat capacity at constant pressure $[\mathrm{J} / \mathrm{kg} / \mathrm{K}]$

$\bar{c}_{p} \quad$ Specific heat capacity at constant pressure $[\mathrm{J} / \mathrm{mol} / \mathrm{K}]$

$f \quad$ LITGS oscillation frequency [Hz]

$\dot{m}$ Mass flow rate [slpm-kg/s]

$p \quad$ Pressure [P]

$p_{\text {atm }} \quad$ Atmospheric pressure $[\mathrm{Pa}]$

$p_{s} \quad$ Partial pressure of the saturated vapour $[\mathrm{Pa}]$

$Q \quad$ Power [W]

$\mathcal{R}$ Gas constant $[\mathrm{J} / \mathrm{mol} / \mathrm{K}]$

$t$ Beam thickness [mm]

$T$ Temperature [K]

$X \quad$ Molar concentration [-]

$X_{S} \quad$ Molar concentration in saturated conditions [-]

$W \quad$ Bulk molecular weight $[\mathrm{g} / \mathrm{mole}]$

$\delta \quad$ Dilution ratio [-]

$\gamma \quad$ Ratio of specific heat [-]

$\lambda$ Laser wavelength [m]

$\Lambda$ Grating spacing [m]

$\rho \quad$ Density $\left[\mathrm{kg} / \mathrm{m}^{3}\right]$

$\sigma_{f} \quad$ Standard deviation of the LITGS frequency $[\mathrm{Hz}]$

$\theta$ Crossing angle (pump beams) [rad]

$\theta_{\mathrm{B}} \quad$ Bragg angle [rad]

$\tau$ Oscillation period [s] 
$\Delta T_{T C} \quad$ Temperature increase measured with thermocouple

$\Delta T_{\text {LITGS }}$ Temperature increase measured with LITGS

[]$_{a b}$ Property of the air + biacetyl gas mixture [-]

[]$_{a}$ Property the air-only flow [-]

[]$_{i}$ Property secondary gas $\left(\mathrm{CO}_{2}, \mathrm{Ar}, \mathrm{He}\right)[-]$

[]$_{0}$ Property of the reference point [-]

\section{INTRODUCTION}

Combustion noise has become a major research interest within the aerospace community. Stricter emission regulations forced the introduction of lean premixed prevaporised combustors, which produce less NOx but burn more unsteadily, generating more noise and creating the potential for combustion instabilities. Fluctuations in the heat release rate of a flame produce isentropic pressure waves (direct noise), as well as pockets of different temperature, density and composition. Once produced, these entropy and composition spots are advected with the mean flow until they reach the first stage of the gas turbine, where they are accelerated, generating sound waves (indirect noise) [1-7]. These acoustic waves are partially reflected upstream in the combustor, where they may couple with the combustor acoustics, triggering a low frequency combustion instability often called rumble [8-10]. The complementary fraction is partially transmitted downstream in the gas turbine, contributing to the overall noise emission [11,12]. Understanding the contribution of indirect mechanisms to combustion noise and instability is an active research area. Studies suggest that they contribute significantly to the core noise [11], but this has not yet been clearly demonstrated in a turbomachinery environment, as there are challenges to source identification by either experimental or numerical methods. Combustion chambers are often described using low-order and simplified models $[4,7,13-16]$, but those might not capture complex geometric and flow characteristics [12,17], which are crucial in determining the amplitude and phase of the entropy-generated sound waves. A major problem consists of the lack of experimental data [11]. A first complication lies in the difficulties in separating direct and indirect noise in the pressure traces acquired in combustors, for which simplified laboratory scale experiments which isolate indirect noise have been designed to test existing analytical and numerical models [18-25]. A second limitation is the lack of information on the dissipation and shear dispersion of the inhomogeneities produced by flames, as few techniques are able to time-resolve unsteady temperature and density variations in combustors. Probes, such as thermocouples, have been used to monitor entropy spots [22], but they suffer from limited time response and may perturb the flow. Non-invasive, high frequency techniques to measure temperature and composition variations in reacting flows include acoustic source-receiver measurements, interferometry, laser absorption spectroscopy, Rayleigh scattering, CARS (Coherent Anti-Stokes Raman Spectroscopy) and LIGS (Laser Induced Grating Spectroscopy). Acoustic source-receiver measurements allow tomographic reconstruction of the temperature field, but involve a complex set-up with many microphones and electrodes connected to a combustor section [26]. Interferometric and laser absorption techniques can achieve good accuracy, but are also limited to line of sight measurements [27,28]. Rayleigh scattering allows high frequency measurements [29], but signals are weak and reflection by surfaces and particles limit the accuracy. Finally, non linear optical techniques, such as CARS and LIGS offer local measurements and good signal to noise ratio. High frequency CARS, however, relies on expensive set-ups, high power lasers and complicated data analysis [30]. LIGS requires a significantly simpler set-up and data analysis than CARS, as has been demonstrated in reacting and non reacting flows to measure gas properties such as temperature, pressure, velocity and species concentration [31-39].

Much of the previous literature has covered LIGS measurements using low repetition rate, high power lasers. Recent experiments have demonstrated LITGS (Laser Induced Thermal Grating Spectroscopy) at frequencies up to $10 \mathrm{kHz}$ [40] at $532 \mathrm{~nm}$ using $\mathrm{NO}_{2}$ as an absorber gas. In the work reported here, high repetition rate LITGS is employed to time resolve the passage of synthetic entropy and composition spots in the Cambridge Entropy Generator [23]. Measurements are performed at 0.5 and $1 \mathrm{kHz}$ using a $355 \mathrm{~nm}$ pulsed laser, using seeded biacetyl to absorb the laser light. To extract density and temperature information from the LITGS signals, a calibration procedure is developed and then validated using a laminar jet, demonstrating that small steady and unsteady changes in the flow composition can be well resolved with LITGS. Finally, LITGS is successfully applied in the Cambridge Entropy Generator to detect the passage of composition and temperature spots, using a quartz tube to visualise the flow. These results suggest that LITGS could be used as a diagnostic technique to time resolve unsteady changes in temperature and composition inside a combustor.

\subsection{MEASUREMENTS OF TEMPERATURE AND COMPOSITION WITH LITGS}

Laser Induced Grating Spectroscopy is akin to a Four Wave Mixing technique based on opto-acoustic and acousto-optic effects arising from the interference of two pulsed laser beams [32]. In a typical LIGS experiment, two parallel polarised, pulsed pump laser beams from the same laser source are crossed at an angle $\theta$ to generate an interference pattern within the 
crossing volume, called a grating, of spacing $\Lambda$, determined by the crossing angle and the laser wavelength $\lambda$ :

$$
\Lambda=\frac{\lambda / 2}{\sin (\theta / 2)}
$$

The interaction between the medium and the radiation in the interference pattern generates, in general, both a resonant and a non-resonant process The non-resonant process arises from an electrostrictive interaction and leads to Laser Induced Electrostrictive Grating Scattering (LIEGS) whereas the resonant process arises from resonant absorption of the radiation energy leading to Laser Induced Thermal Grating Scattering (LITGS). The electrostrictive interaction involves the polarizability of the molecules such that they are driven towards the regions of lower electric field intensity. The resulting pressure perturbation drives two oppositely propagating acoustic waves which form a standing acoustic wave with a spatially periodic density perturbation oscillating with time at a frequency determined by the transit time of the sound waves across the grating period $[32,34,41]$. The resonant process requires that the wavelength of the incident pump light correspond to a spectral absorption in some molecular species in the gas. Molecular excitation is higher in corresponding regions of high field intensity in the interference (grating) pattern. The absorbed energy is subsequently released by collisional quenching processes, resulting in localised perturbations to the temperature and density in the form of a thermal grating. The latter modulation is accompanied by associated pressure and density waves, which interact periodically in and out of phase with the temperature grating, which can be detected as a locally varying field. The matter of which of the resonant or non-resonant processes dominates depends upon the properties of the incoming light and the species present in the interaction region. In absorbing media the thermal grating process dominates, leading to LITGS signals which can be observed using relatively low energy pulses (1 $10 \mathrm{~mJ})$ [38]. Electrostrictive gratings, on the other hand, do not require an absorbing species, and so can be induced by non-tunable lasers, but, since they are normally several orders of magnitude weaker than the thermal gratings, they require much higher energy pulses $(10100 \mathrm{~mJ})[32,38]$.

Although the LITGS technique offers significantly higher signal to noise than LIEGS, its main limitation is the need for sufficient concentrations of a resonant species at the available wavelength. On the other hand, both techniques benefit from the higher densities available in experiments at higher pressures. At present there is not a sufficient database of experiments at pressure to identify which species and at what concentrations are required.

A probe beam incident at the appropriate Bragg angle $\theta_{\mathrm{B}}$ to the grating generates the scattered a beam, which encodes the frequency modulating the grating density oscillation.

$$
\theta_{\mathrm{B}}=\sin ^{-1} \frac{\lambda}{2 \Lambda}
$$

The propagating wave in the grating moves at the local speed of sound $c$, so that the modulation of the scattered signal has been shown to appear at a frequency determined as:

$$
f=n \frac{c}{\Lambda}=2 n \frac{c}{\lambda} \sin \frac{\theta}{2}
$$

where $n=1$ for thermal gratings and $n=2$ for electrostrictive gratings. The local temperature or composition can be obtained from this frequency of oscillation of the LITGS signal, assuming an ideal gas equation of state, as

$$
c^{2}=\frac{1}{n^{2}} f^{2} \Lambda^{2}=\frac{\gamma \mathcal{R} T}{W}=\frac{\gamma p}{\rho}
$$

where $\gamma$ is the ratio of specific heats, $\mathcal{R}$ the universal gas constant, $W$ the bulk molecular weight of the mixture, and $p$, $T$ and $\rho$ the local pressure, temperature and density. Equation 4 shows that the measured frequency is proportional to the local speed of sound, and can therefore be related to perturbations in temperature, pressure or density if the thermodynamic properties of the bulk mixture are known. Conversely, if the mixture is at a constant temperature, variations in thermodynamic properties (and thus the gas concentration) can be determined. In this paper, the LITGS technique is used to probe both aspects of flow fluctuations. Equation 4 refers only to the information that can be extrapolated from the frequency of the oscillations. However, it has been demonstrated that information about the pressure and composition can also be inferred from the contrast and the decay rate of the LITGS signals [42], which might be useful to evaluate the contribution of temperature or composition variations in complex flows when these change simultaneously. 


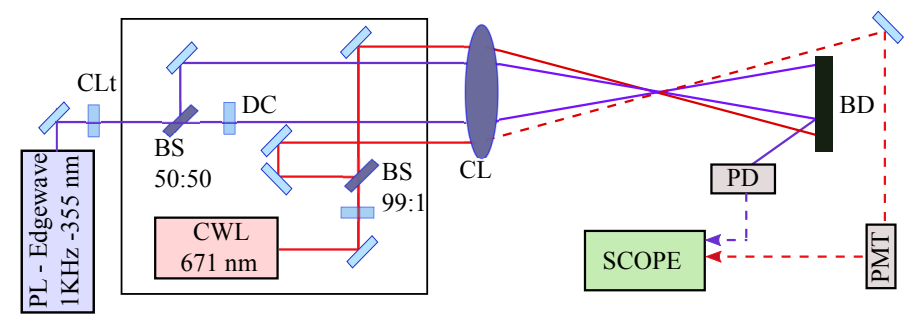

Fig. 1: Optical layout of the experiment. PL: $355 \mathrm{~nm}$ pulsed edgewave laser; CWL: continuous solid state laser; PD: photodiode; PMT: photomultiplier; HRM: highly reflective mirror; CLT: converging lens for telescopic arrangement; BS: beam splitter; BD: beam dump; CL: crossing lens; DC: delay compensator plate; S: signal; T: trigger

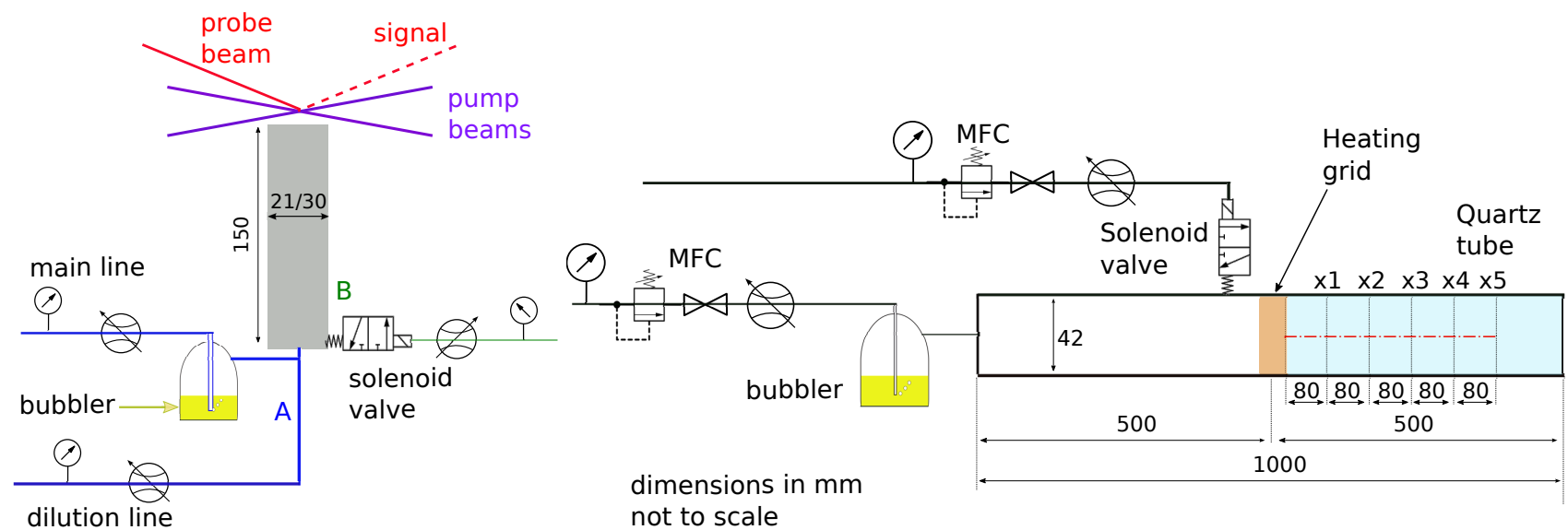

Fig. 2: Left: schematic of the jet experiment set-up. Right: schematic of the Entropy Wave Generator with the optical access window

\section{EXPERIMENTAL SET-UP}

\subsection{OPTICAL LAYOUT}

The optical layout of the experiment is sketched in Fig. 1. An Edgewave IS200-2-L laser generates pulsed laser beams with a wavelength $\lambda=355 \mathrm{~nm}$, delivering $0.5-4 \mathrm{~mJ}$ per pulse. The frequency of the laser can be varied and in the present experiments the frequencies are between 0.5 and $1 \mathrm{kHz}$. A 50/50 beam splitter divides the $355 \mathrm{~nm}$ beam into two identical beams. A $12 \mathrm{~mm}$ thick quartz plate compensates for the optical delay between the two pump beams. The probe volume is defined by the region intersected by both pump beams as well as the the probe beams in a forward folded boxcar arrangement [43]. The two pump beams, separated by $12 \mathrm{~mm}$, are crossed by a $300 \mathrm{~mm}$ focal length crossing lens, resulting in a crossing angle $\theta \simeq 2.3^{\circ}$, producing a grating of spacing $8.7 \mu \mathrm{m}$ in the probe volume with length and width of approximately $50 \mathrm{~mm}$ by $1 \mathrm{~mm}$. The beams in the probe volume are collimated by a telescopic arrangement of lenses rather than allowing the crossing lens to also focus the beams at the crossing point. This has two benefits. Firstly, the diameter of the interaction region is kept large enough to ensure that the signal does not decay as a result of the transit of the acoustic waves away from the probed volume, so the signal oscillations are maintained within the time limited only by the diffusion and viscous damping effects. Secondly, this arrangement produces a collimated signal beam that can be propagated to the detector efficiently without requiring a re-collimating lens near the interaction region where it is prone to collect scattered light that reduces the signal-to-noise ratio. [39]. The continuous probe beam is produced by a CNI MLL-III-671 diodepumped solid state laser $(\lambda=671 \mathrm{~nm}$, power $=300 \mathrm{~mW}$, diameter $\sim 1 \mathrm{~mm})$ and sampled via a 99:1 splitter. The scattered signal is detected using a Hammamatsu photomultiplier (H6780-20). A photodiode (DET210) is used to provide a trigger signal for the data acquisition using pump light scattered from the beam dump. The signals are recorded using a 4-channel LeCroy 6104A oscilloscope (sampling rate $10 \mathrm{Gs} / \mathrm{s}, 1 \mathrm{GHZ}$ bandwidth, 40 million points maximum real time memory) in sequential mode, for $1 \mu \mathrm{s}$ (10000 points) per signal. Oscilloscope memory limitations mean that a maximum of 4000 shots can be sequentially recorded and stored. Once the memory buffer is filled, a few seconds are needed to store the buffer and start a new acquisition. 


\subsection{EXPERIMENTAL LAYOUT}

The experiments are run in two different configurations (Fig. 2). An open laminar jet is used for calibrating the technique, while an Entropy Generator Rig (similar to the one described in [23]) with optical access is used to detect the passage of entropy and composition spots in a weakly turbulent flow.

The laboratory air supply system provides filtered air, and Alicat mass flow controllers regulate the flow: two 250 slpm ( $\pm 0.8 \%$ accuracy, $\pm 1 \%$ full scale) and one $20 \mathrm{slpm}$ ( $\pm 0.8 \%$ accuracy, $\pm 1 \%$ full scale). The mean flow is seeded with biacetyl (2,3-Butanedione; $99 \%$; Acros Organics CAS: 431-03-8) using two Dreschel bottle bubblers with sintered heads (a 0.251 bottle for flow rates lower than $20 \mathrm{slpm}$ and a 0.51 bottle for higher flow rates). The bubblers are kept at ambient temperature, and deliver nearly saturated mixtures of biacetyl, which are diluted downstream. Density and mixture fraction variations are induced in the air flow by adding carbon dioxide, argon or helium. The gases are pulsated into the tube using a fast response solenoid valve (Asco Numatics HSM2L7H50V) located in line with a $50 \mathrm{slpm}$ Alicat mass flow controller ( $\pm 0.8 \%$ accuracy, $\pm 1 \%$ full scale). The valve is activated using a relay driven by a signal generator while powered by a separate power supply (Glassman Lp 60-20).

Table 1: Properties of air, carbon dioxide, argon, helium and biacetyl

\begin{tabular}{clllll}
\hline & Air & $\mathrm{CO}_{2}$ & $\mathrm{Ar}$ & $\mathrm{He}$ & Biacetyl \\
\hline$W[\mathrm{~g} / \mathrm{mol}]$ & 28.97 & 44.01 & 39.948 & 4.002 & 86.09 \\
$\bar{c}_{p}[\mathrm{~J} / \mathrm{mol} / \mathrm{K}]$ & 29.115 & 37.144 & 20.78 & 20.77 & 120.97 \\
\hline
\end{tabular}

\subsubsection{Laminar jet}

The laminar air jet (Fig. 2a), consisting of a $21 \mathrm{~mm}$ diameter, $150 \mathrm{~mm}$ long stainless steel tube, is used for calibration (30 $\mathrm{mm}$ diameter jet for temperature calibration). The LITGS beams cross about $2 \mathrm{~mm}$ above the tip of the tube. The main gas line which passes through the bubbler is connected to a secondary dilution line 1 meter downstream of the bubbler. The jet tube has two ports: the air line (bubbler and dilution) is connected at port A. The second port B is used for the pulsated flow. An in-line heater (AHP-7562 Omega Engineering, $240 \mathrm{~V}, 750 \mathrm{~W}, 140 \mathrm{~mm}$ heated length) can be added to the flow line for higher temperatures.

\subsubsection{Entropy tube}

Entropy and composition spots are generated via pulsed power to a heating grid [23] or pulsed injection from a secondary flow [24]. The duct is adapted from the Entropy Generator [23], to provide optical access to the flow downstream of the entropy source. The system is positioned on a 3-axes traverse, driven by three stepper motors controlled via a Labview interface. The LITGS probe is aligned to the centreline of the duct at different locations, marked 1 to $5 \mathrm{in}$ (Fig. $2 \mathrm{~b}$ ).

Filtered compressed air from the laboratory air supply system is fed to the $250 \mathrm{slpm}$ Alicat mass flow controller, which is connected to the 0.51 bubbler via an $8 \mathrm{~mm}$ internal diameter plastic tube. An $8 \mathrm{~mm}$ diameter, 1.5 meter long hose connects the bubbler with the system through a flange. The duct is 1 meter long, divided into two parts, clamped together via metal flanges. The first part houses the pulsating valve and heating grid and consists of a $500 \mathrm{~mm}$ long PVC tube of $42.6 \mathrm{~mm}$ inner diameter. The secondary flow is injected $450 \mathrm{~mm}$ downstream of the inlet, connecting the solenoid valve to the rig via a 200 $\mathrm{mm}$ long, $5 \mathrm{~mm}$ inner diameter plastic hose. The heating grid is held in place using a metal cavity upstream of the optically accessible section. The latter consists of a $42 \mathrm{~mm}$ inner diameter, $500 \mathrm{~mm}$ long UV grade fused silica tube (wall thickness of $2 \mathrm{~mm}$ ). Two metal flanges hold the quartz tube in place, and two $500 \mathrm{~mm}$ long rods reinforce the structure. The downstream end of the rig is left open to the atmosphere.

\section{RESULTS: MIXTURE FRACTION AND TEMPERATURE MEASUREMENTS}

In this section, we investigate how accurately LITGS frequency measurements can be converted into temperature or mixture fraction fluctuations. For the calibration experiments, a flow of $5 \mathrm{slpm}$ of air with saturated biacetyl forms a laminar jet (Fig. 2a), whilst the LITGS probe volume is located at the centerline of the vertical jet at about $2 \mathrm{~mm}$ from the tube rim. A total of 1000 shots of 10000 points at $10 \mathrm{Gs} / \mathrm{s}$ are acquired. The raw voltage signal is padded with zeros up to $2^{19}$ samples (approximately 52 times longer) prior to taking its Fast Fourier Transform. The frequency of the oscillation corresponds to 


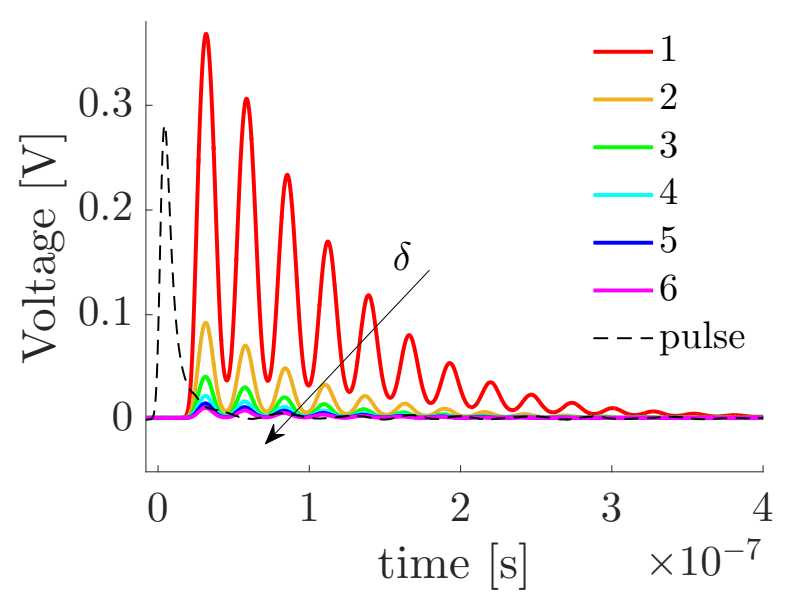

(a)

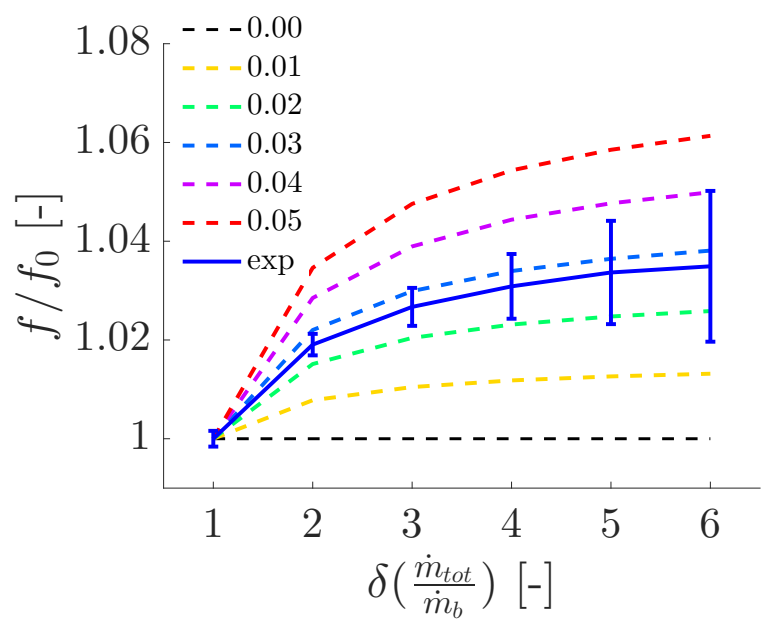

(b)

Fig. 3: (a) Ensemble averaged LITGS time traces obtained by increasing the dilution ratio $\delta=\dot{m}_{t o t} / \dot{m}_{b}$; (b) Frequency peaks for each dilution ratio, normalised by the frequency at zero dilution. Blue solid line: experimental data; dashed lines: values predicted for a given molar concentration of saturated undiluted biacetyl $X_{v}$. Black line: normalised litgs frequency for $X_{b}=0$ (no biacetyl in the mean flow).

the highest peak in the power spectrum. In what follows, the standard deviation in the frequency is denoted as $\sigma_{f}$, and is shown as the error bars in the experimental plots.

\subsection{TRADE OFF BETWEEN INTRUSIVENESS AND PRECISION}

LITGS is based on the generation of a local density change by absorption of energy, and is therefore slightly intrusive, unless the product of pump beam energy and the concentration of the absorber are kept low [44, 45]. On the other hand, the signal itself, proportional to the square of the density perturbation [37], needs to be sufficiently high so as to provide suitable signal to noise for an assessment of the frequency. This might require the addition of a non negligible quantity of an resonant species to the mean flow and the use of sufficiently high pump laser energy, which can potentially perturb the flow characteristics. To avoid thermalisation effects in the probe volume, the laser fluence must be kept low. A compromise must be achieved between increasing the diameters of the beams at the crossing point to obtain low thermalisation, and the need for spatial resolution in the longitudinal direction. To avoid the change in the mean flow properties due to the absorber, low concentration of a seeding species with properties similar to the mean flow should be used.

In this section we show how seeding the mean flow with an absorber in trace amounts affects the mean flow properties and therefore the extracted LITGS signal frequency. Figure 3 shows how variations in the dilution ratio of the biacetyl stream cause a decrease of the amplitude (a) and shift in the frequency (b) of LITGS signals. A constant mass flow rate of 5 slpm (approximately $0.1 \mathrm{~g} / \mathrm{s}$ ) flows through the bubbler. This main stream is diluted by adding extra air with the dilution line downstream of the bubbler, for dilution ratios $\delta$ between the total mass flow rate and the bubbler flow rate as $\delta=\dot{m}_{t o t} / \dot{m}_{b u b}$ 
between 1 (undiluted) and 6. Figure 3 a shows that the signals decrease ins strength from the undiluted to the most diluted mixture, as expected from the proportionality with the density change by absorption. The experimental plot (solid line) of the frequency $f$ obtained for the signal at each dilution ratio, normalised by the undiluted conditions $f_{0}$ is shown in Fig. 3b. The measured peak frequency increases with increasing dilution, and so do the corresponding standard deviations. The change in frequency (gradient of the plot) decreases with increasing dilution, until the values reach an approximately asymptotic value.

Clearly one wishes to have high signals and low standard deviations, but with a single value for the frequency, representing the expected behaviour with zero added biacetyl to the mixture and no temperature increase in the probe volume due to the laser. An optimum dilution ratio must be used for which the signal is sufficiently strong, the standard deviation sufficiently low, the energy of the pump laser does not increase the probe volume temperature and any corrections due to the changes in properties produced by the tracer biacetyl must be taken into account. The following development explains the underlying theory to resolve the issues.

The expected ratio between measured frequencies for two different dilution ratios $\delta$ can be calculated by expressing the quantities in Eq. 4 as a function of the biacetyl concentration $X_{b}$, and the expected local temperature rise by absorption, $\Delta T$.

$$
f^{2}=\frac{1}{\Lambda^{2}} \frac{\gamma \mathcal{R} T}{W}=\frac{1}{\Lambda^{2}} \frac{\left(1-\frac{\mathcal{R}}{\bar{c}_{p, a}\left(1-X_{b}\right)+\bar{c}_{p, b} X_{b}}\right)^{-1} \mathcal{R}}{W_{a}\left(1-X_{b}\right)+W_{b} X_{b}}\left(T_{a}+\Delta T\right)
$$

Assuming negligible thermalisation effects $\left(\Delta T \simeq 0\right.$ ), the expected frequency $f_{a}$ for the pure jet of air is obtained if either $X_{b} \rightarrow 0$ (which leads to a poor quality of the signal) or for absorbers with an identical specific heat and molecular mass as the mean flow values (which is not the case of biacetyl, as shown in Table 1).

Accurate measurements of the molar fractions of biacetyl $X_{b}$ in the flow after the bubbler are challenging in the absence of absolute standards. However, from the knowledge of the vapour pressure of biacetyl at a given bubbler temperature $p_{s}(T)$, the ideal maximum concentration of biacetyl in saturated conditions can be estimated as $X_{b, s}=p_{s} / p_{0}$, where $p_{0}$ is the total pressure in the bubbler. From [46] the vapour pressure of biacetyl at room temperature is $p_{s}=0.058$ bar, thus $X_{b, s, \max }=0.058$. A calibration procedure is introduced to estimate the actual molar concentration of biacetyl in the mean flow, and the necessary correction to the frequency.

For the laser fluences characteristic of the current experiment, it can be demonstrated (not shown here) that the temperature increase of the probe volume due to the thermalisation induced by the laser is negligible ( $\Delta T \rightarrow 0$ in Eq. 5), so the ratio of frequencies between two biacetyl mole fractions can be expressed as:

$$
\left(\frac{f_{\delta 2}}{f_{\delta 1}}\right)^{2}=\frac{\left(1-\frac{\mathcal{R}}{\bar{c}_{p, a}\left(1-X_{\delta 2}\right)+\bar{c}_{p, b} X_{\delta 2}}\right)^{-1}}{\left(1-\frac{\mathcal{R}}{\bar{c}_{p, a}\left(1-X_{\delta 1}\right)+\bar{c}_{p, b} X_{\delta 1}}\right)^{-1}} \frac{W_{a}\left(1-X_{\delta 1}\right)+W_{b} X_{\delta 1}}{W_{a}\left(1-X_{\delta 2}\right)+W_{b} X_{\delta 2}}
$$

where the total molar fraction of biacetyl $X_{\delta}$ for each dilution ratio $\delta$ is:

$$
X_{\delta}=\frac{X_{b}}{\left(1-X_{b}\right) \delta+X_{b}}
$$

The dashed lines in figure $3 \mathrm{~b}$ show the frequency ratio $f_{2} / f_{1}$ calculated from Eq. 6 by varying $X_{b}$. The value $X_{b}=0.028$ solves Eq. 6 . The black line shows the limit case where no biacetyl is added to the flow $\left(X_{b}=0\right)$, so an increase in the total mass flow rate does not change the bulk flow properties.

Figure $3 \mathrm{~b}$ shows that even for low biacetyl concentrations $\left(X_{b}=0.01\right)$, the frequency change for saturated conditions relative to no dilution $\left(\Delta f_{\max } / f_{0}=2 \%\right)$ is not negligible. However, in order to obtain high signal to noise ratios and low standard deviations, a relatively high concentration of biacetyl is required. Thus, the change in the mean flow properties due to the addition of biacetyl must be accounted for. Such a high variation of the mean flow properties due to biacetyl addition is caused by the substantial difference in the specific heat and molecular weight between air and biacetyl, as shown in Table 1. In this experiment, the use of biacetyl as the absorbing molecule is forced by the wavelength of the pump laser: to the authors' knowledge, biacetyl is the only semi-harmless volatile substance which absorbs the $355 \mathrm{~nm}$ wavelength to generate the thermal grating. The maximum energy delivered from the pulses of the laser $(4 \mathrm{~mJ})$ is not sufficient to obtain detectable electrostrictive signals. In this study we aim to demonstrate the principle that laser induced thermal gratings can be obtained with high frequency lasers, obtaining low standard deviations in the generated signals. In a real combustor, LITGS can 


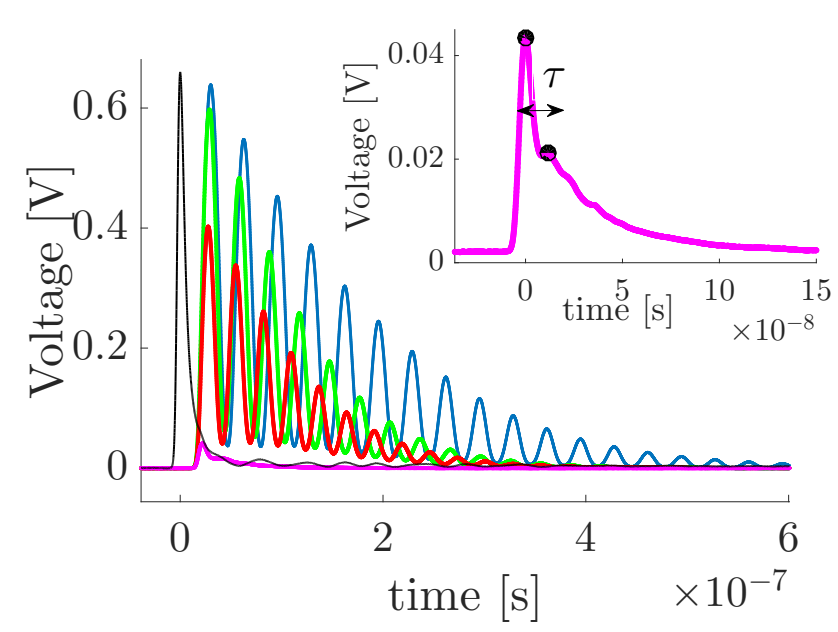

(a)

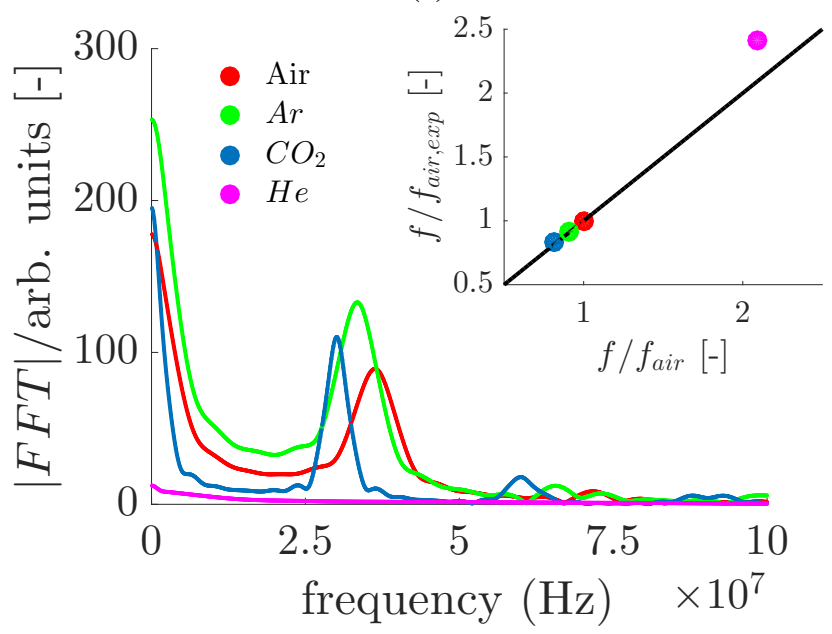

(b)

Fig. 4: (a) Ensemble-averaged litgs signals acquired in a jet of biacetyl-saturated air, Argon, Carbon dioxide and Helium. Inset: zoom on helium signal. (b) Corresponding Fourier Transform of the signals. Inset: ratio between the peak frequencies obtained with different gases, normalized by that with air

potentially be a suitable technique to detect unsteady temperature and composition changes in the flow by using a pump laser wavelength tuned to an absorption line of a species in the mixture. Candidates in the reactant side exist for quadrupled Nd:YAG frequencies $266 \mathrm{~nm}$ (e.g. toluene, which is also present in the fuel), or even oxygen in the deeper UV excimer range $(172 \mathrm{~nm})$. In the absence of absorbing species, high power lasers can be used to generate electrostrictive gratings, from which temperature and composition can be detected as well. As the strength of the signal increases with the fourth power of the pressure for LITGS and with the square power of the pressure with LIEGS, these techniques are suitable candidates to be used in pressurised gas turbines.

\subsection{RELATIVE SPEED OF SOUND IN DIFFERENT GASES}

In this section, we deploy LITGS to measure the changes in the speed of sound in different gases, using pure jets of air, carbon dioxide, argon and helium seeded with biacetyl. Mass flow rates of $5 \mathrm{slpm}$ of air, $\mathrm{CO}_{2}$, $\mathrm{Ar}$ and $10 \mathrm{slpm}$ of $\mathrm{He}$ flow through the $250 \mathrm{ml}$ bubbler with no further dilution.

Figure 4 shows the ensemble averaged LITGS signals in the four gases, in the time (Fig. 4a) and in the frequency domain (Fig. 4b). The speed of sound in $\mathrm{CO}_{2}$ and $\mathrm{Ar}$ is lower than in air, so the corresponding oscillation frequencies are lower. For helium, however, the speed of sound is much higher, and, as the quenching efficiency is low, the amplitude of the LITGS signal in helium is weak compared to the other gases, and only two peaks could be observed in time (Inset in Fig. 4a). Therefore, in this specific case of pure helium, the corresponding frequency can not be extracted from the frequency spectrum, as it is mainly flat (Fig. 4b), and it is instead estimated from the interval between peaks as $f=\frac{1}{\tau}$ from the averaged 

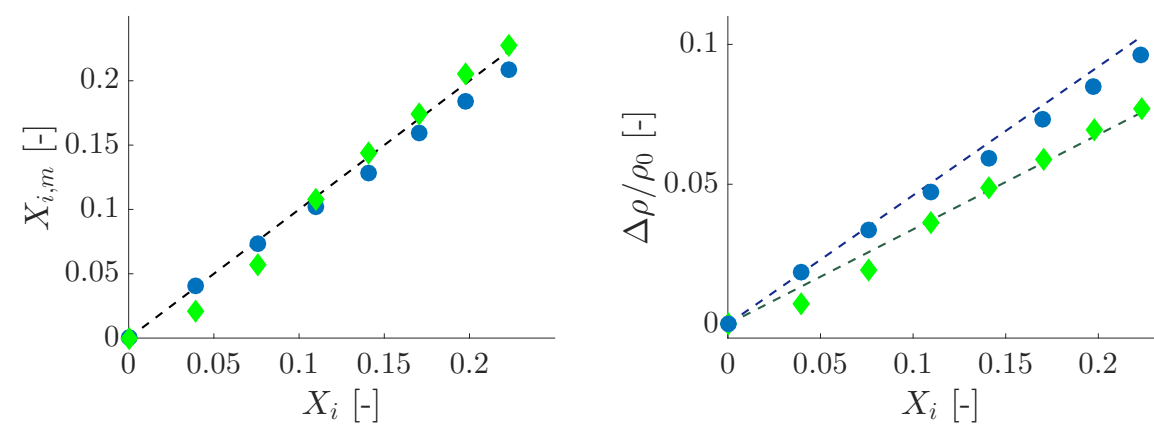

Fig. 5: (left) Measured mean molar concentrations in a jet of air of $\mathrm{CO}_{2}$ (blue dots) and Argon (green diamonds) and (right) mean density variations using LITGS, relatively to the expected concentrations based on dilution ratios

signal. In the inset of figure $4 \mathrm{~b}$, the theoretical oscillation frequency of the gases normalised by that of air frequency $f_{0}$ are compared with the experimental values (a molar concentration of biacetyl $X_{b}=0.03$ is included in the calculation). Good agreement is found between predictions and experimental data. The higher error obtained with helium is probably due to the large difference in molecular mass between biacetyl and helium, for which the diffusivity of biacetyl in helium is lower and therefore the concentration of biacetyl in the jet of pure helium is lower than in the other gases.

\subsection{STEADY COMPOSITION VARIATIONS}

LITGS can also be used detect relatively small changes in the composition of a flow under steady conditions (Fig. 5). A secondary flow of $\mathrm{CO}_{2}$ and $\mathrm{Ar}$ is steadily added (port $\mathrm{B}$, no solenoid valve) to the base laminar jet flow (Fig. 2a with undiluted vaporised air and biacetyl flow, port A). The flow at the measurement point is assumed to be homogeneous, given the long mixing length relatively to diameter. The addition of the secondary flow changes both the specific heat capacity and the molecular mass of the flow passing through the probe volume. The molar concentration of the secondary gas $X_{i}$ is calculated using:

$$
\left(\frac{f}{f_{0}}\right)^{2}=\frac{\left(1-\frac{\mathcal{R}}{\bar{c}_{p, i} X_{i}+\bar{c}_{p, 0}\left(1-X_{i}\right)}\right)^{-1}}{\left(1-\frac{\mathcal{R}}{\overline{\bar{c}_{p, 0}}}\right)^{-1}} \frac{W}{W_{i} X_{i}+W\left(1-X_{i}\right)}
$$

where the base flow of air and biacetyl (0) is used as a reference. The density variation in the mean flow due to the addition of the secondary flow is computed as

$$
\frac{\Delta \rho}{\rho}=\frac{\Delta W}{W}=\frac{W_{i} X_{i}+W\left(1-X_{i}\right)}{W}-1
$$

These results show that changes in the mean flow properties can be detected if the determination of the LITGS frequency is sufficiently precise.

\subsection{TEMPERATURE MEASUREMENTS}

In what follows we derive a calibration procedure to correctly evaluate the temperature increase from the LITGS data. A base flow of 40 slpm of air passes through the bubbler (no dilution), is warmed up by the in-line resistance heater by varying the applied voltage, and forms a jet in the $30 \mathrm{~mm}$ tube. A thermocouple is used to monitor the temperature increase, using a flow of $40 \mathrm{slpm}$ of pure air (no biacetyl) while the resistance warms up until the temperature stabilises, to reduce the biacetyl consumption. The data recorded with the thermocouple (dashed line in Fig. 6) refers to air-only case. Once the recorded temperature is stable, the same mass flow rate of air is passed through the bubbler and the heater and LITGS traces are recorded over the tracer-laden laminar jet, at the same location where the thermocouple was located. Once the LITGS signal is recorded, the air flow is switched from the bubbler and the voltage on the resistance is increased, repeating the same procedure for the next temperature acquisition. The temperature increase $\Delta T_{a b}$ in the air and biacetyl flow (magenta circular markers in Fig. 6) is obtained from the LITGS oscillation frequency as: 


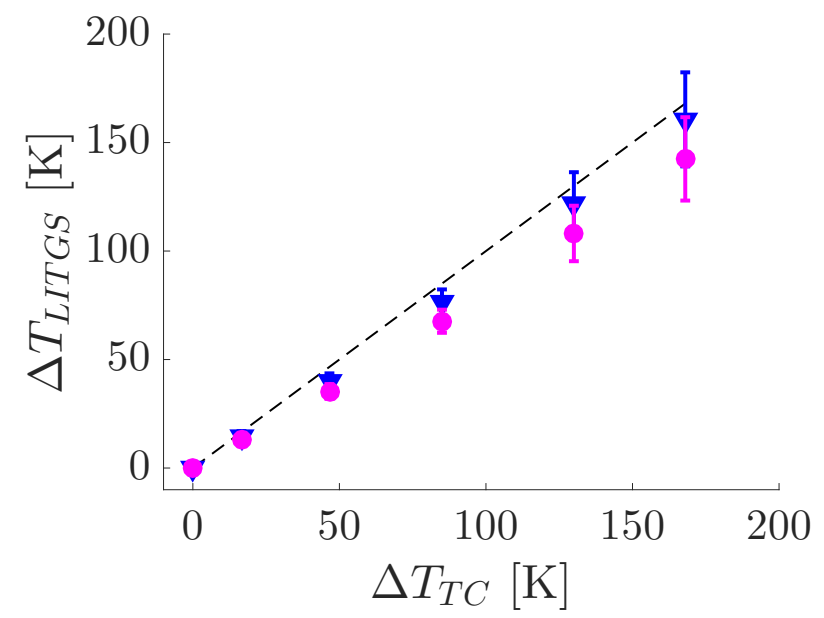

Fig. 6: Measured temperature rise from ambient using LITGS, plotted against the thermocouple measurements. Dashed line: thermocouple measurements in the pure air jet, magenta dots: litgs measurement in the air and biacetyl jet, blue dots: equivalent temperature increase in the pure air jet from the LITGS measurements

$$
\Delta T_{a b}=\left[\left(\frac{f_{a b}}{f_{0}}\right)^{2}-1\right] T_{0}
$$

where $T_{0}=294 \mathrm{~K}$ is the ambient temperature and $f_{0}$ is the corresponding frequency. Part of the discrepancy between the LITGS and the thermocouple measurements can be explained in the different molecular mass and specific heat of the flows with and without biacetyl. The heater delivers a constant power $Q$ to the flow, so the relation between the temperature increase in the flow with $\left(\Delta T_{a b}\right)$ and without $\left(\Delta T_{a}\right)$ biacetyl is

$$
\frac{\Delta T_{a}}{\Delta T_{a b}}=\frac{\frac{Q}{\bar{c}_{p, a} \dot{m}_{a}}}{\frac{Q}{\bar{c}_{p, a b} \dot{m}_{a b}}}=\left(1+\frac{X_{b}}{1-X_{b}} \frac{\bar{c}_{p, b}}{\bar{c}_{p, a}}\right)
$$

The measured temperature rise in the mixture with air only would be higher than that measured with air plus biacetyl for a given heating power. Once this factor is accounted for, the two measurements agree within $2 \%$ (blue symbols in Fig. 6). For high temperature variations, the temperature dependency of $c_{p}$ should be also taken into account in Eq. 11 .

\section{TIME RESOLVED COMPOSITION VARIATION MEASUREMENTS IN A LAMINAR JET}

Since we demonstrated that LITGS can resolve the difference between different speeds of sound, it is possible to use it as a marker of the mixture fraction in a binary mixture, if the molecular weights of the mixing streams are significantly different. In this section we show that LITGS can time resolve perturbations in the density and composition of an air jet gas generated by unsteady pulses of argon, carbon dioxide and helium. A steady mass flow rate of 24 slpm of air is used as base flow in the laminar jet (2b): half of the flow passes through the bubbler and the other half through the dilution line, for a mean flow velocity $u=1.15 \mathrm{~m} / \mathrm{s}$ and a $\mathrm{Re}=1558$. Periodic spots of the alternative gas are injected at the bottom of the jet (port B), using the fast response valve. The frequency of the pulses is set to $3 \mathrm{~Hz}$, with a duty cycle of $20 \%$. The pressure in the gas tank is regulated to about 3 bar. The LITGS signal is acquired at $1 \mathrm{kHz}$. Figure 7a shows the time-resolved (non averaged) LITGS frequency traces acquired within $1 \mathrm{~s}$, corresponding to three cycles of the valve, normalised by the base flow frequency $f_{0}$. The shape and amplitude of the gas pulses can be clearly identified in the frequency traces, including the time characteristics of the rise and decay time. While the pulses of carbon dioxide and argon are well defined, the helium pulses are more spread out at the end of the pulse, when the highest quantity of helium is injected in to the flow, causing a shorter signal with a smaller number of oscillations, as shown in Fig. 4. Thus, the helium data are interpolated with an interpolation envelope determined using the RMS of 30 consecutive points. The molar fraction $X_{i}$ of the injected gas corresponding to each LITGS frequency is determined using Eq. 8, and plotted in 7b. The volumetric flow rates injected 

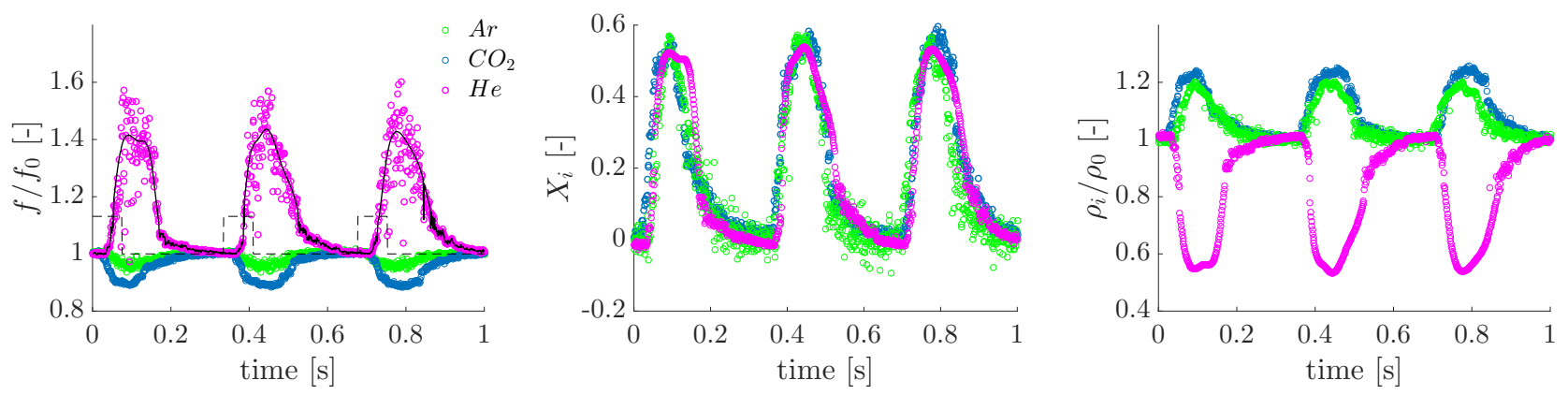

Fig. 7: (a) Normalised time resolved frequency variation obtained from LITGS measurements in a laminar jet pulsated with a secondary gas; (b) Corresponding time resolved molar fraction of the pulsated gas ; (c) Corresponding relative measured density variation

by the valves for the three gases are similar for the different gases, as expected. From the molar fraction $X_{i}$, the density variation at each point is obtained from Eq. 9. As the density of helium differs substantially from the air, the variation of density after the helium pulses is much higher than for $\mathrm{CO}_{2}$ and Ar.

\section{TIME RESOLVED MEASUREMENTS IN AN ENTROPY GENERATION DUCT}

The aim of the calibration and validation measurements reported in the previous sections is to enable accurate unsteady measurements of concentration and entropy spots through a duct, so as to map out their convection and dispersion. Here these are demonstrated along the optically accessible cylindrical section described in Fig. 2b. The small diameter of the cylinder poses a particular problem when aligning the beams into the quartz tube, as small out of plane misalignments of the rig can easily deflect the three beams towards different directions whereas they need to cross at exactly the same point, particularly as the probe beam enters the probe volume at an angle with respect to the horizontal plane. The duct height is adjusted using the traverse until the centerline of the cylinder is coplanar with the the two pump beams. A few iterations were required to maintain the crossing point between the two pump and one probe beams along the same plane across the cylinder centreline. The larger the radius of curvature, the less severe is this problem.

The mass flow rate of air in the experiment is set to $80 \mathrm{slpm}(1.57 \mathrm{~g} / \mathrm{s})$, which corresponds to an upstream velocity of about $1 \mathrm{~m} / \mathrm{s}$ and a Reynolds number $\mathrm{Re}=2800$, so the flow is in a nearly turbulent regime. Nearly saturated air flows are used, with low or no dilution, and this is the main factor limiting the flow rates. The maximum velocities are limited by the consumption rate of biacetyl and the volume of the bubbler: relatively high mass flow rates lead to lower biacetyl molar fractions, yet flow rates cannot be increased without causing spillages of biacetyl. An alternative vaporisation method or a laser wavelength tuned to a more strongly-absorbing region of the molecular spectrum would allow higher flow rates to be achieved.

Under these conditions, using $0.5 \mathrm{~mJ}$ of energy in the pump beams, a typical base flow signal has an average amplitude of $200 \mathrm{mV}$, versus the $350 \mathrm{mV}$ in the open jet. The standard deviation over 1000 points in the LITGS signal of the base flow is $\sigma_{f}=3.5 \times 10^{5}$, so $\sigma_{f} / f_{0}=1 \%$. This corresponds to a standard deviation of $\sigma_{T}=5 \mathrm{~K}$. For a higher pump beam energy of $4 \mathrm{~mJ}$, the amplitude of the signal becomes $400 \mathrm{mV}$, and the standard deviation decreases to $\sigma_{f} \simeq 10^{5}$, so $\sigma_{f} / f_{0}<0.3 \%$. This corresponds to a standard deviation of $\sigma_{T} \simeq 1 \mathrm{~K}$.

The laser pulse frequency in the present tests is limited $500 \mathrm{~Hz}$, so as to allow the acquisition of longer periods of time in the data set due to the limited space in the memory buffer. No difference is found regarding either the laser pulses shapes and amplitudes, or in the signal characteristics (mean frequency and standard deviation) between the higher and lower pulse frequencies used.

\subsection{DETECTION OF COMPOSITION SPOTS}

Composition spots are detected using LITGS in the quartz tube at the five centreline positions indicated in Fig. 2. The different gases $\left(\mathrm{CO}_{2}, \mathrm{Ar}, \mathrm{He}\right)$ are injected at a frequency of $1 \mathrm{~Hz}$ and a duty cycle of $20 \%$. At each point, the passage of about 30 spots is recorded, then the rig is moved from one place to the other using the traverse.

Figure 8 (First row) shows the instantaneous time resolved traces at five of the locations for $\mathrm{CO}_{2}$, Ar and He pulses. The passage of spot the can be clearly identified in these signals. In order to decrease the standard deviation and obtain more accurate measurements, the energy of the laser pulses is set to $4 \mathrm{~mJ}$. It was experimentally verified that negligible temperature perturbation from absorbed energy was observed with this level of energy in the pumps, but the standard deviation decreases substantially, leading to more accurate measurements. The consumption of biacetyl level in the bubbler generates a small 

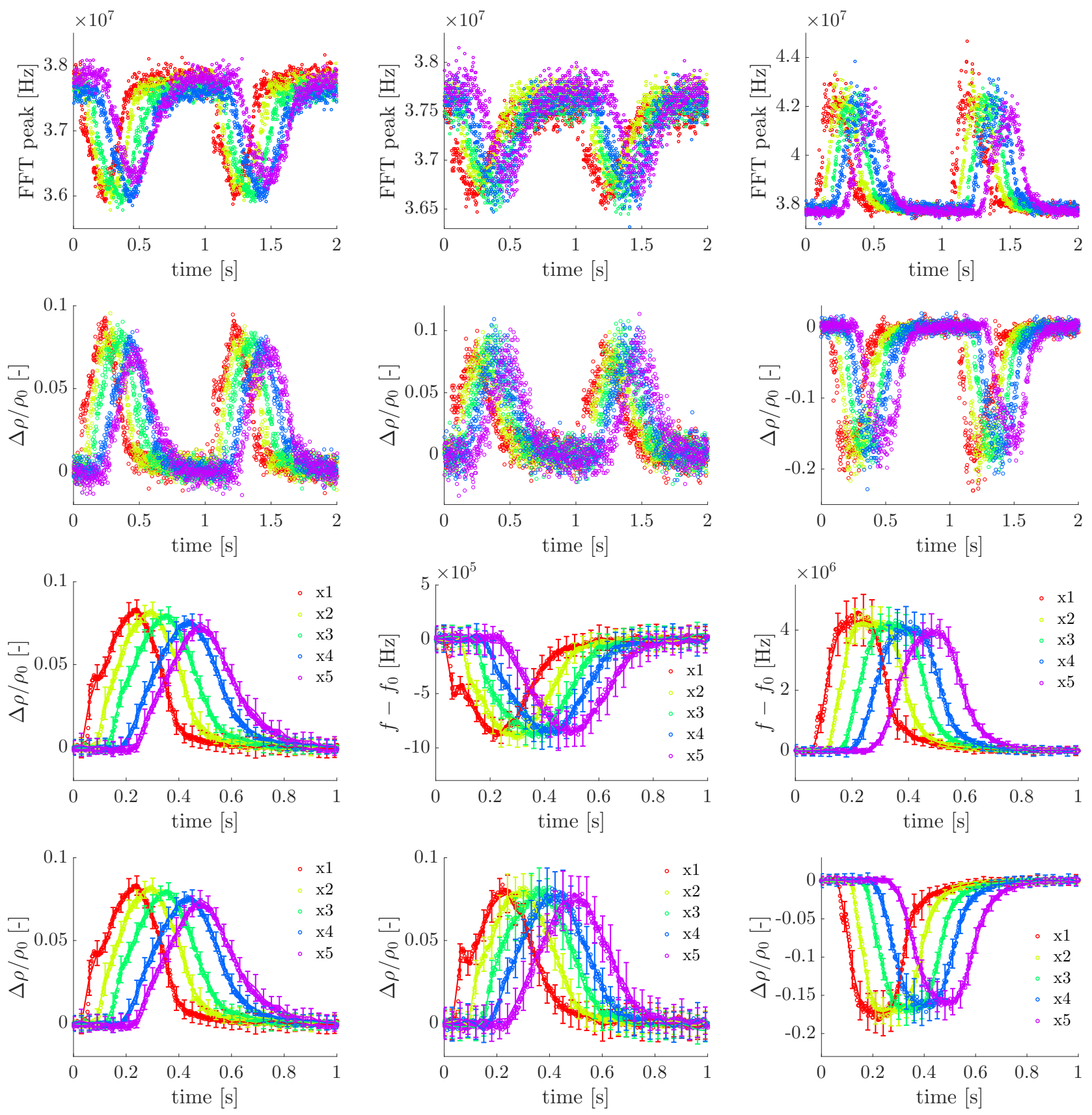

Fig. 8: Detection of of composition spots (from left to right column: $\mathrm{CO}_{2}, \mathrm{Ar}, \mathrm{hHe}$ ) at five locations along the quartz tube. Injection pulse frequency: $1 \mathrm{~Hz}$, duty cycle: $20 \%$. Rows: 1) time resolved LITGS traces (peak frequency); 2) time resolved normalised density variations, 3) ensemble-averaged (30 pulses) LITGS traces (frequency variation from the mean); 4) ensemble-averaged (30 pulses) normalised density variations

shift of the base flow frequencies. As the level of biacetyl in the bubbler drops, the vaporisation becomes less efficient, the concentration of biacetyl decreases and the corresponding mean flow frequency increases (Fig. 3). The change in concentration has to be taken into account to properly evaluate the mean flow properties at each location. The mean flow has a dilution $\delta=1.3$, and the molar concentration of biacetyl in the point with the lowest mean flow frequency (which corresponds to the highest biacetyl concentration) is find to be $X_{b}=0.013$, with the procedure described in section 3.1. The biacetyl concentration for the other flow rates is then evaluated from the mean flow frequency using Eq.8 and 9 .

Once the mean flow properties for each location are determined, the mole fraction and density variation of the pulsed gas is determined using Eq. 5. An error in the evaluation of the biacetyl concentration in the mean flow of $\Delta X_{b}=0.01$ (e.g. if $X_{b}=0.04$ instead) would lead to an error in the density of the injected flow $\Delta \rho_{i} / \rho_{i}<1 \%$, thus the quantitative results are reliable. The 30 composition spot pulses are phase averaged to obtain clearer information on the shape and amplitude 

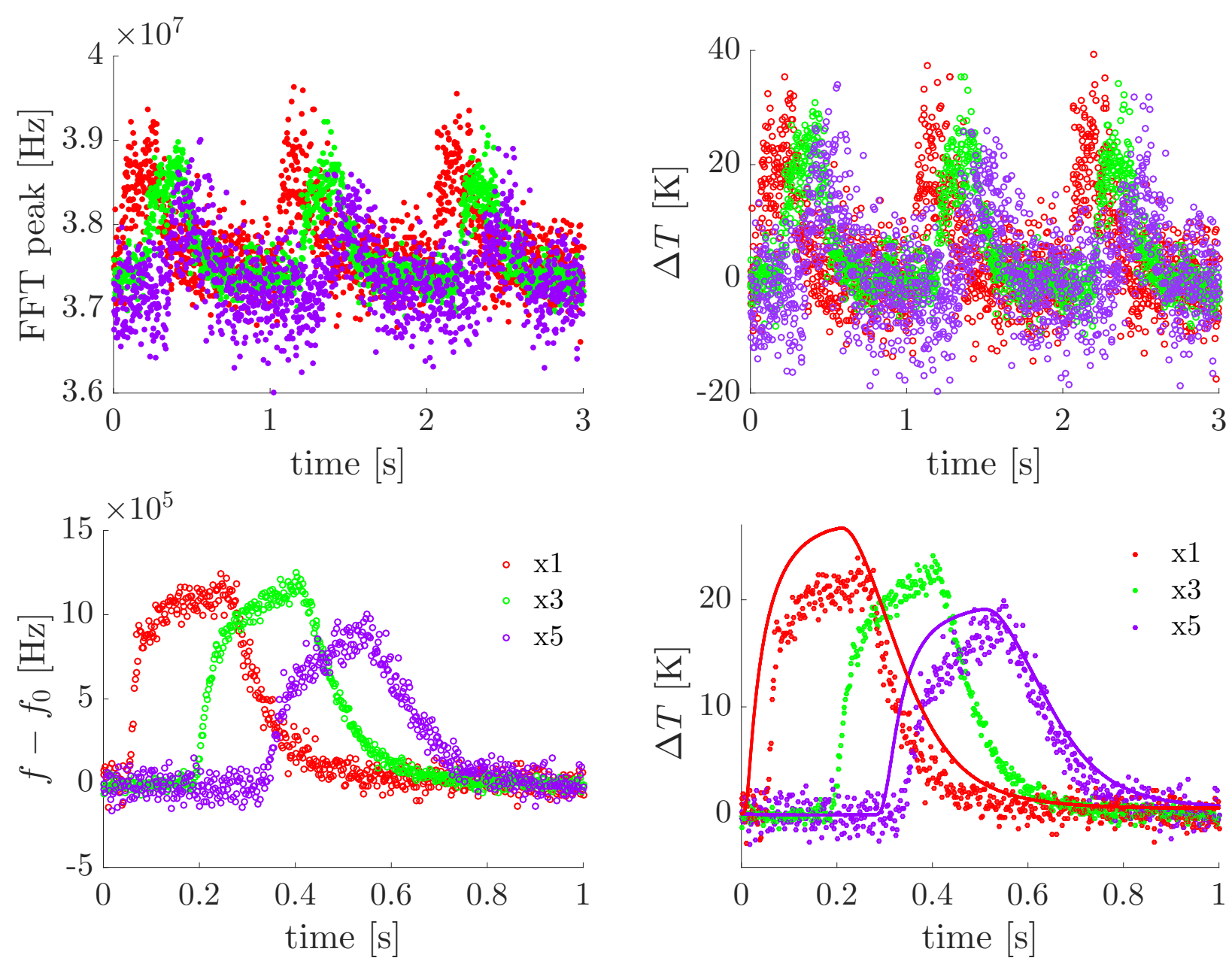

Fig. 9: Measured time-resolved temperature fluctuations: (a) frequency; (b) temperature deviation from initial value, (c) averaged frequency variation, (d) ensemble-averaged temperature obtained with LITGS (circles) and with anemometer and thermocouples (lines) from [23]

of the spots, reducing the scatter of the measurements (third and fourth raws). However, the features of the injected pulses can be observed directly from the instantaneous measurements. For example, the signals acquired in position $x 1$ display a small hump in the first few milliseconds, which corresponds to the first bounce of the composition spot off the opposite wall after being injected, a feature which disappears in the following positions. Some effects of dispersion can be observed in the pulses, despite the short distance within the five measurements locations. The spots at $x 1$ have a sharper shape, while at $x 5$ they are more spread out. For the helium, as expected, the signals are more noisy, relatively to $\mathrm{CO}_{2}$ and Ar. However, it can be observed that diffusive effects have a higher impact on helium than on $\mathrm{CO}_{2}$ and $\mathrm{Ar}$, as helium is a smaller and lighter molecule, with a higher diffusion coefficient.

These preliminary experiments demonstrate that LITGS can be used to detect the unsteady composition variations of a gas mixture in a turbulent flow. As not many techniques are available, this may help the validation of diffusion-dispersion models. In a combustor or a gas turbine, time resolved measurements of unsteady temperature and composition changes may allow to quantify the strength and amplitude of the entropy and composition spots surviving through the chamber and entering into the nozzle, and help to quantify the indirect noise generated from their acceleration. The main barrier to achieving this at the moment is finding a good match between available species and laser wavelength.

\subsection{DETECTION OF TEMPERATURE SPOTS}

This section describes the results of LITGS measurements applied to detect imposed temperature variations on the flow via a heating grid, and advected and dispersed along the flow, where they are acquired with LITGS at locations $x 1, x 3$ and $x 5$ in Fig. 2b. The experimental conditions for this experiment are chosen to be comparable to the experimental case $\mathrm{C} 1$ reported in [23], in order to compare the LITGS results with the temperature data previously acquired with thermocouples and anemometers. Pulses of time length $\tau=200 \mathrm{~ms}$ and input power $35 \mathrm{~V} \times 21 \mathrm{~A}$ on the power supply are delivered into 
the heating grid. The mass flow rate and duct dimensions are chosen to be similar: here, a mass flow rate of 80 slpm (with dilution $\delta=1$ ) runs into the $42 \mathrm{~mm}$-inner-diameter rig; in [23], a mass flow rate of $78 \mathrm{slpm}$ flowed into the $42.6 \mathrm{~mm}$-innerdiameter rig. The temperature measurements are acquired at similar locations. In [23], the temperature of the entropy spot at the grid and $40 \mathrm{~mm}$ downstream are reported to be $\Delta T=26.6$ and $21.1 \mathrm{~K}$, respectively.

Figure $9 a$ shows the time resolved frequency traces obtained with LITGS. To reduce the scatter, the signal is averaged over 30 phase-locked traces (Fig. 9b). After taking the base flow frequency shift into account, the temperature increase $\Delta T$ is determined using the calibration procedure described in Sec. 3.4 (Fig. 9b) The averaged temperature traces obtained in this way are compared with the measurements in [23]. The comparisons between the thermocouple and LITGS measurements are very favourable, even though the measurements are acquired in different facilities with different flow rates at slightly different positions. In [23] the thermocouple measurements are corrected using a faster hotwire, and some inaccuracy is also expected, of the order of 1-2 K. Overall, the rise and fall of the temperature measurements is very well captured, and the differences should be attributed to differences in the experimental setup, including the fact that the heating grid losses may be slightly different in the two setups, as the pipe materials varied. More importantly, it should be noted that there are few techniques capable of detecting temperature variations of such small magnitude at high frequencies. In the present work the upper limit of the measurement frequency has not been explored owing to the limitation of the repetition rate of the laser available. However, it would appear that these measurements are possible at rates exceeding $1 \mathrm{kHz}$ if a suitable laser is available [40].

\section{CONCLUSION}

For the first time LITGS has been applied to make time-resolved measurements of temperature and composition variations $(<3 \%)$ in a non-reacting flow. A low pulse energy, $\mathrm{kHz}$ repetition rate laser is used to generate the grating, obtaining high quality and precise measurements, with standard deviations of the order of $1 \%$. As LITGS is a relatively simple technique which requires limited optical access to the measurement volume and offers simple data analysis, it is a good candidate to detect unsteady temperature and composition variations in combustion chambers. However, this study highlights some of the limitations of the technique. The main complication lies in the requirement of an absorbing species in the flow at the specific wavelength of the laser, which may be achieved by seeding the flow, thus altering the mean flow properties. The second disadvantage is the trade off between perturbing the temperature by use of tightly focussed beams and degrading the spatial resolution. Larger beam diameters are desirable to obtain a longer residence time of the acoustic perturbation within the measurement volume and to minimize temperature perturbations caused by local heating effects arising from absorption in high laser fluence. However, a trade-off is demanded, since it is important also to minimize the longitudinal extent of the measurement volume to avoid probing regions of different temperature and to achieve the highest possible spatial resolution.

In this experiment, biacetyl is used as a tracer in the flow to absorb the $355 \mathrm{~nm}$ laser light and generate the thermal grating. It is demonstrated that the addition of biacetyl generates non-negligible property changes to the local mixture. A calibration procedure is developed to evaluate the concentration of biacetyl, and thus recover the temperature and composition from the oscillation frequency. A further validation experiment in unsteady conditions demonstrates that LITGS could time resolve unsteady perturbations. Finally, LITGS is successfully applied to detect the passage of entropy and composition spots in an enclosed quartz duct. This suggests LITGS as a suitable technique to analyse the generation, dissipation and dispersion of entropy spots. These measurements should allow the quantification of the strength and amplitude of the entropy and composition spots surviving through the chamber and entering into the nozzle, and help to quantify the indirect noise generated from their acceleration.

\section{Acknowledgements}

Francesca De Domenico is supported by the Honorary Vice-Chancellor's Award and a Qualcomm/ DTA Studentship (University of Cambridge). Experiments were partly funded by EPSRC grant EP/K02924X/1, and the high frequency laser was funded by EP/K035282/1. The authors gratefully acknowledge Erwan O. Rolland for his help with the traverse and solenoid valve operation, and Dr. Lee Weller for his technical advice and help with the set-up.

\section{References}

[1] Chu, B. T., and Kovasznay, L. C. G., 1958. "Non-linear interactions in a viscous heat-conducting compressible gas". Journal of Fluid Mechanics, 3, pp. 494-514.

[2] Morfey, C. L., 1973. "Amplification of Aerodynamic Noise by Convection Flow Inhomogeneities.”. Journal of Sound and Vibration, 31(4), pp. 391-397.

[3] Ffowcs Williams, J. E., and Howe, M. S., 1975. "The generation of sound by density inhomogeneities in low Mach number nozzle flows". Journal of Fluid Mechanics, 70(03), pp. 605-622. 
[4] Marble, F., and Candel, S., 1977. "Acoustic disturbance from gas non-uniformities convected through a nozzle". Journal of Sound and Vibration, 55(2), pp. 225-243.

[5] Cumpsty, N. A., 1979. "Jet engine combustion noise: Pressure, entropy and vorticity perturbations produced by unsteady combustion or heat addition". Journal of Sound and Vibration, 66(4), pp. 527-544.

[6] Howe, M. S., 2010. "Indirect combustion noise". Journal of Fluid Mechanics, 659, pp. 267-288.

[7] Magri, L., O’Brien, J., and Ihme, M., 2016. "Compositional inhomogeneities as a source of indirect combustion noise". Journal of Fluid Mechanics, 799, pp. R4 1-12.

[8] Polifke, W., Paschereit, C. O., and Döbbeling, K., 2001. "Constructive and destructive interference of acoustic and entropy waves in a premixed combustor with a choked exit". Journal of Acoustics and Vibration, 6(3), pp. 135-146.

[9] Goh, C. S., and Morgans, A. S., 2013. "The influence of entropy waves on the thermoacoustic stability of a model combustor". Combustion Science and Technology, 2(185), pp. 249-268.

[10] Hochgreb, S., Dennis, D., Ayranci, I., Bainbridge, W., and Cant, S., 2013. "Forced and self-excited instabilities from lean premixed, liquid-fuelled aeroengine injectors at high pressures and temperatures". In Proceedings of the ASME Turbo Expo 2013: GT2013-95311, pp. 1-12.

[11] Dowling, A. P., and Mahmoudi, Y., 2015. "Combustion Noise". Proceedings of the Combustion Institute, 35(1), pp. 65-100.

[12] Ihme, M., 2017. "Combustion and Engine-Core Noise". Annual Review of Fluid Mechanics, 49, pp. 277-310.

[13] Dowling, A. P., and Stow, S. R., 2003. "Acoustic analysis of gad turbine combustors". Journal of Propulsion and Power, 19(5), pp. 751-764.

[14] Goh, C. S., and Morgans, A. S., 2011. "Phase prediction of the response of choked nozzles to entropy and acoustic disturbances". Journal of Sound and Vibration, 330(21), pp. 5184-5198.

[15] Moase, W., Brear, M., and Manzie, C., 2007. "The forced response of choked nozzles and supersonic diffusers". Journal of Fluid Mechanics, 585, pp. 281-304.

[16] Duran, I., and Moreau, S., 2013. "Solution of the Quasi One-Dimensional Linearized Euler Equations using Flow Invariants and the Magnus Expansion". Journal of Fluid Mechanics, 723, pp. 190-231.

[17] Motheau, E., Nicoud, F., Mery, Y., and Poinsot, T., 2013. "Analysis and Modelling of Entropy Modes in a Realistic Aeronautical Gas Turbine". Journal of Engineering for Gas Turbines and Power, 135(September 2013), p. V01AT04A009.

[18] Bohn, M. S., 1976. "Noise produced by the interaction of acoustic waves and entropy waves with high speed nozzle flows". PhD thesis, California Insitute of Technology.

[19] Bake, F., Richter, C., Mühlbauer, B., Kings, N., Röhle, I., Thiele, F., and Noll, B., 2009. "The Entropy Wave Generator (EWG): A reference case on entropy noise". Journal of Sound and Vibration, 326(3-5), pp. 574-598.

[20] Gaetani, P., Persico, G., and Spinelli, A., 2015. "Entropy wave generator for indirect combustion noise in a highpressure turbine". In Proceedings of the 11th European Conference on Turbomachinery Fluid Dynamics and Thermodynamics.

[21] Knobloch, K., Werner, T., and Bake, F., 2015. "Noise generation in hot nozzle flow". In Proceedings of the ASME Turbo Expo 2015: GT2015-43702GT2015-43702, pp. 1-14.

[22] Giusti, A., Worth, N. A., Mastorakos, E., and Dowling, A. P., 2017. "Experimental and Numerical Investigation into the Propagation of Entropy Waves". AIAA Journal, 55(2).

[23] De Domenico, F., Rolland, E. O., and Hochgreb, S., 2017. "Detection of direct and indirect noise generated by synthetic hot spots in a duct". Journal of Sound and Vibration, 394, pp. 220-236.

[24] Rolland, E. O., De Domenico, F., and Hochgreb, S., 2017. "Theory and application of reverberated direct and indirect noise". Journal of Fluid Mechanics, 819, pp. 435-464.

[25] Tao, W., Schuller, T., Huet, M., and Richecoeur, F., 2017. "Coherent entropy induced and acoustic noise separation in compact nozzles". Journal of Sound and Vibration, 394, pp. 237-255.

[26] Wassmer, D., Schuermans, B., Paschereit, C. O., and Moeck, J. P., 2017. "Measurement and modeling of the generation and the transport of entropy waves in a model gas turbine combustor". International Journal of Spray and Combustion dynamics, 9(4), pp. 299-309.

[27] Eckbreth, A., 1996. Laser diagnostics for combustion temperature and species Vol.3. CRC Press.

[28] Hanson, R. K., and Davidson, D. F., 2014. "Recent advances in laser absorption and shock tube methods for studies of combustion chemistry". Progress in Energy and Combustion Science, 44, pp. 103-114.

[29] Rausch, A., Fischer, A., Konle, H., Gaertlein, A., Nitsch, S., Knobloch, K., Bake, F., and Rohle, I., 2011. "Measurements of Density Pulsations in the Outlet Nozzle of a Combustion Chamber by Rayleigh-Scattering Searching Entropy Waves". Journal of Engineering for Gas Turbines and Power, 133(3), p. 031601.

[30] Roy, S., Gord, J. R., and Patnaik, A. K., 2010. "Recent advances in coherent anti-Stokes Raman scattering spectroscopy : Fundamental developments and applications in reacting flows". Progress in Energy and Combustion Science, 36(2), pp. 280-306.

[31] Eichler, H., Gunter, P., and Pohl, D., 1986. Laser-Induced Dynamic Gratings. Springer Berlin Heidelberg. 
[32] Cummings, E. B., 1994. "Laser-induced thermal acoustics : simple accurate gas measurements". Optics Letter, 19(17), pp. 1361-1363.

[33] Paul, P. H., Farrow, R. L., and Danehy, P. M., 1995. "Gas-phase thermal contributions to four-wave mixing". Journal of the Optical Society of America, 12(3), pp. 384-392.

[34] Stampanoni-Panariello, A., Kozlov, D. N., Radi, P. P., and Hemmerling, B., 2005. "Gas phase diagnostics by laserinduced gratings I . theory". pp. 101-111.

[35] Latzel, H., Dreizler, A., Dreier, T., Heinze, J., Dillmann, M., Stricker, W., Lloyd, G. M., and Ewart, P., 1998. “Thermal grating and broadband degenerate four-wave mixing spectroscopy of $\mathrm{OH}$ in high-pressure flames". Applied Physics B, 673, pp. 667-673.

[36] Walker, D. J. W., Williams, R. B., and Ewart, P., 1998. “Thermal grating velocimetry". Optics Letters, 23(16), pp. 1316-1318.

[37] Stevens, R., and Ewart, P., 2004. "Single-shot measurement of temperature and pressure using laser-induced thermal gratings with a long probe pulse". Applied Physics B, 117, pp. 111-117.

[38] Sander, T., Altenhöfer, P., and Mundt, C., 2014. "Development of Laser-Induced Grating Spectroscopy for Application in Shock Tunnels". Journal of Thermophysics and Heat Transfer, 28(1), pp. 27-31.

[39] Williams, B., and Ewart, P., 2012. "Photophysical effects on laser induced grating spectroscopy of toluene and acetone". Chemical Physics Letters, 546, pp. 40-46.

[40] Förster, F. J., Crua, C., Davy, M., and Ewart, P., 2017. "Time resolved gas thermometry by laser induced grating spectroscopy with a high repetition rate laser system". Experiments in Fluids, 58(7), pp. 1-8.

[41] Cummings, E. B., Leyva, I. A., and Hornung, H. G., 1995. "Laser-induced thermal acoustics (LITA) signals from finite beams". Applied Optics, 34(18), pp. 3290-3302.

[42] Kiefer, J., Kozlov, D. N., Seeger, T., and Leipertz, A., 2008. "Local fuel concentration measurements for mixture formation diagnostics using diffraction by laser-induced gratings in comparison to spontaneous Raman scattering". Journal of Raman Spectroscopy, 39(April), pp. 711-721.

[43] Eckbreth, A. C., and Anderson, T. J., 1986. "Simultaneous rotational coherent anti-Stokes Raman spectroscopy and coherent Stokes Raman spectroscopy with arbitrary pump-Stokes spectral separation". Optics Letters, 11(8), pp. 496498.

[44] Willman, C., and Ewart, P., 2016. "Multipoint temperature measurements in gas flows using 1 D laser induced grating scattering". Experiments in Fluids, 57(12), pp. 1-9.

[45] Lowe, S. M., 2018. "Quantitative measurements of temperature using laser-induced thermal grating spectroscopy in reacting and non-reacting flows". $\mathrm{PhD}$ thesis, University of Cambridge.

[46] Neely, W., and Hall, T., 1972. "Vapor Pressure of Biacetyl". Journal of Chemical and Engineering Data, 17(3). 